\title{
Identification and Analysis of Hazardous Materials Using Optical Spectroscopy
}

\author{
Surik Khudaverdyan', Vazgen Meliqyan', Taron Hovhannisyan', \\ David Khudaverdyan', Ashok Vaseashta ${ }^{2,3}$ \\ ${ }^{1}$ National Polytechnic University of Armenia, Yerevan, Armenia \\ ${ }^{2}$ International Clean Water Institute, Manassas, VA, USA \\ ${ }^{3}$ Institute of Electronic Engineering and Nanotechnologies “D. GHITU”, Academy of Sciences of Moldova, Chisinau, Moldova \\ Email: xudaver13@mail.ru
}

How to cite this paper: Khudaverdyan, S., Meliqyan, V., Hovhannisyan, T., Khudaverdyan, D. and Vaseashta, A. (2017) Identification and Analysis of Hazardous Materials Using Optical Spectroscopy. Optics and Photonics Journal, 7, 6-17. http://dx.doi.org/10.4236/opj.2017.71002

Received: December 14, 2016

Accepted: January 19, 2017

Published: January 22, 2017

Copyright $\odot 2017$ by authors and Scientific Research Publishing Inc. This work is licensed under the Creative Commons Attribution International License (CC BY 4.0).

http://creativecommons.org/licenses/by/4.0/

(c) (i) Open Access

\begin{abstract}
This investigation is aimed towards using optical spectroscopy for remote identification and quantitative analysis of hazardous substances for safety and security applications. We introduce a new model employing portable photosensor devices that are based on the double-barrier and vertically placed silicon structure, for such applications. The different absorption depths of individual waves allow us to carry out their spectral selection using an algorithm developed for this specific objective. We tested the proposed model on experimental Ag-p-Si-n-Si structures. The algorithm is developed for the spectral analysis without the preliminary calibration. The low dark currents (several dozens of $\mathrm{pA}$ ) permit us to carry out the spectral analysis of the integral flux of the electromagnetic radiation of low intensity. The quantitative data from light current-voltage characteristics allow us to obtain an intensity distribution spectrum characteristic of the material by using red LED and the green laser. The results of this investigation divulge new possibilities for the creation of a new type of the portable semiconductor spectrophotometer and due to its stand-off detection capability, offer potential pathways to evaluate hazardous substances.
\end{abstract}

\section{Keywords}

Distribution Spectrum, Photosensors, Optical Spectroscopy, Radiation Intensity

\section{Introduction}

In recent years, increasing demand for the stand-off detection/sensing for hazardous chemical has significantly increased due to their use in safety, security, environmental protection and detect suspicious packages, wire, fragmentation 
materials, or other signs of improvised explosive devices (IEDs) or crisis situations. Standoff techniques enable detection of threats without contact. The possibility of contamination (for operators and/or equipment) is thus avoided, and the need for subsequent decontamination is eliminated. In addition, standoff detection of explosives allows identification of threats from safe distances, i.e., outside the blast zone. There are now ongoing efforts to extend these methodologies to even biological threats. It is, hence of paramount importance to increase the efficiency of such methods and solution pathways which will respond quickly to such crisis situations. It is particularly important to create user-friendly sensors platform which will likely enable the remote analysis of the different materials in different media.

Remote spectrophotometric sensors are of special interest these days, as they provide us with necessary information on the composition of the mediupm under investigation and hence solve the omnipresent problem of identification from the security stand-point [1] [2] [3] [4] [5]. Nevertheless, the information received from studies of natural objects by existing means is not satisfactory from the point of their quantitative and qualitative analysis. There is a significant gap in terms of dispersion of the spectral data of the identical objects even in the advanced methods of investigation of the optical spectral analysis, which often excludes correlation between the measurement results. The processing of the spectral data is obtained by quantitative analysis and the reliability of such results depends directly on the parameters of the device in use.

It is critical that several sensors are required for monitoring large areas. To optimize cost and to strategically place sensors in regions which are likely to provide a fair assessment of the contamination scenario, it is urgent to develop; a): inexpensive and small-size sensors with high spectral sensitivity, suitable for remote field identification, and, b): appropriate algorithms for accurate registration of data measured by these sensors. In methods that are currently available for spectral analysis, the spectral distribution of the electromagnetic radiation is obtained by light filters, a prism, a diffraction grating and high accuracy mechanical devices [6] [7] [8]. The spectrophotometric systems like this are not multi-purpose and require additional devices and external software support for the fulfillment of every new function. Thus, they are rather expensive and are not suitable for field deployment.

The effective solution to the above-stated problems is by development of a semiconductor structure in which the electronic processes will provide high accuracy spectral analysis of the electromagnetic radiation. Several researchers have conducted study using multi-colored photoreceivers [9]-[17] in which multilayer structures or a cascade chain of active layers with different base thicknesses are used. In such photoreceivers, the different penetration depths of individual waves of the radiation provide different degrees of photoconductivity. The mathematical modeling of the measurement results provides information on the spectral distribution of the intensity. The registration accuracy of structures mentioned above is essential due to the necessity to having identical ab- 
sorption conditions with nanometric accuracy for multiple photodiodes and multilayer structures. The complexity involved during fabrication of photodiodes and multilayer structures during their development and the difficulty in controlling the spectral sensitivity by the external voltage impedes their development and usage. The present investigation offers a viable means of high level of accuracy during spectral analysis with the help of the electronic processes in $\mathrm{n}^{+}-\mathrm{p}-\mathrm{n}^{+}$structures.

\section{Device Structure}

The structure under investigation is a silicon structure, as prepared using standard integrated circuit processing methods. The structure consists of an $n^{+}-\mathrm{p}-\mathrm{n}^{+}$ structure (Figure 1). The p-base is occupied by the depleted layers of two barriers (Figure 1). The current through the structure is the difference of the currents of the two oppositely directed potential barriers. The position of the point of contact of the depleted layers $\mathrm{x}_{\mathrm{m}}$ (Figure 1 ) depends on the polarity of the external voltage and is moved to the surface of the forward biased $n^{+}-p$ junction (ohmic contact). The electromagnetic radiation is incident on the left ohmic contact as shown in Figure 1. The registration area of the radiation is in the depleted layers. In presence of applied voltage, the reverse biased $n^{+}$-p junction is dominating and thus it's depleted layer becomes wider. In the meantime, the depleted layer of the forward biased junction becomes thinner. As a result, the current is mainly governed by the reverse biased $\mathrm{p}-\mathrm{n}^{+}$junction. The incident electromagnetic waves have different penetration depths with respect to their wavelengths and intensities.

Since the shift of $x_{m}$ towards the surface (towards "0" in the Figure 1) widens the range of current generation, different waves contribute according to their wavelength. The thin $\mathrm{p}^{+}$layer towards the left surface with high conductivity can be neglected, the "0" point can be taken as the surface, and the voltage drop at the front contact can be neglected. This allows us to measure of the photocurrent which contains information with respect to their wavelengths and intensities.

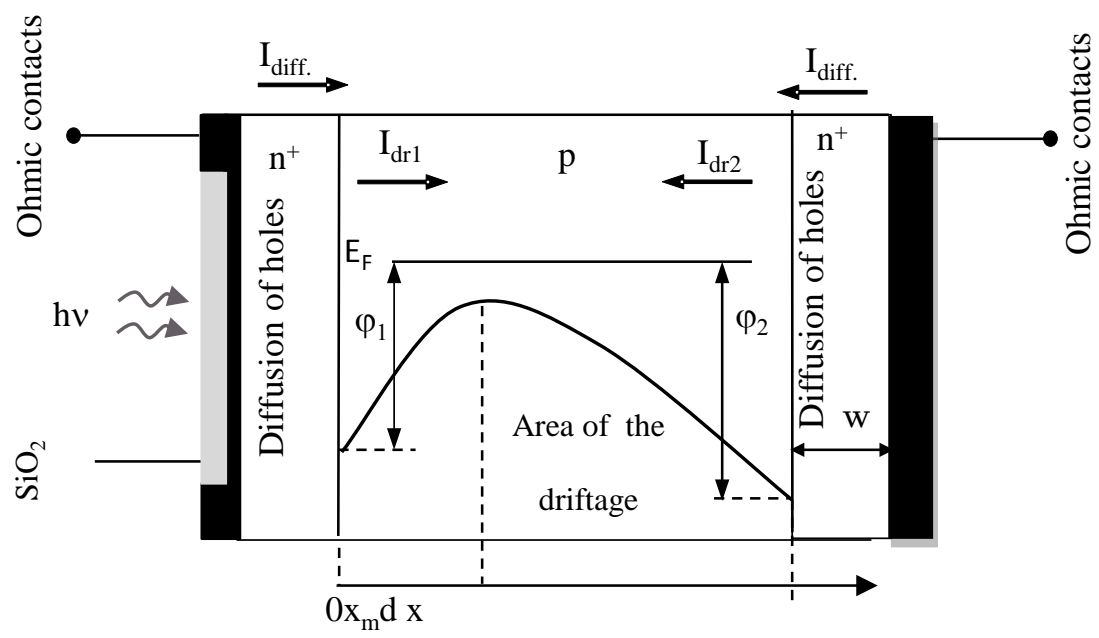

Figure $1 . n^{+}-p-n^{+}$structure and the directions of currents in it. 
The information can be obtained by a relevant algorithm. For the development of such algorithm, it is necessary to find the relationship between $x_{m}$, applied voltage $V$, absorption coefficient $\alpha$ and incident intensity $F$. Considering that the base is covered by the depleted regions of both barriers, we determine the distribution of potential in the spatial charge region of the double-barrier structure, as shown in Figure 2. For this, we solve the Poisson equation which connects the field potential $V(x)$ with the volume density of the charges creating the field,

$$
\frac{d^{2} V(x)}{d x^{2}}=-\frac{\rho}{\varepsilon \varepsilon_{0}}
$$

In Poisson's equation, the potential $V(x)$ can be related to the potential energy of electrons $\varphi(x)$, as, $(\varphi(x)=-q V(x))$.

Since $\rho=q N_{a}$, we obtain,

$$
\frac{d^{2} \varphi}{d x^{2}}=\frac{N_{a} q^{2}}{\varepsilon \varepsilon_{0}}
$$

where $N_{a}$ is the concentration of acceptor impurities, $\varepsilon$ is the relative dielectric permeability of the substance, $\varepsilon_{0}$ is the dielectric permeability of the vacuum, and $q$ is the electron charge.

The boundary conditions for the given equation are $\left(\left|\frac{d \varphi}{d x}\right|=0\right)$ at $x=x_{m}$ (Figure 2, $x_{m}$ is the minimum of the potential energy of electrons) and $\varphi(x)=\varphi_{1}$ at $x=0$ (Figure 1).

Taking into account the boundary conditions, we integrate Equation (2) and obtain,

$$
\varphi(x)=\frac{q^{2} N_{a}}{2 \varepsilon \varepsilon_{0}} x^{2}-\frac{q^{2} N_{a}}{\varepsilon \varepsilon_{0}} x_{m} x+\varphi_{1}
$$

When $x=d \quad$ (Figure 2).

$$
\varphi(x)=\frac{q^{2} N_{a}}{2 \varepsilon \varepsilon_{0}} d^{2}-\frac{q^{2} N_{a}}{\varepsilon \varepsilon_{0}} x_{m} d+\varphi_{1}=\varphi_{2}+q V
$$

It is possible to find the dependences of $x_{m}$ and $d-x_{m}$ on the external voltage:

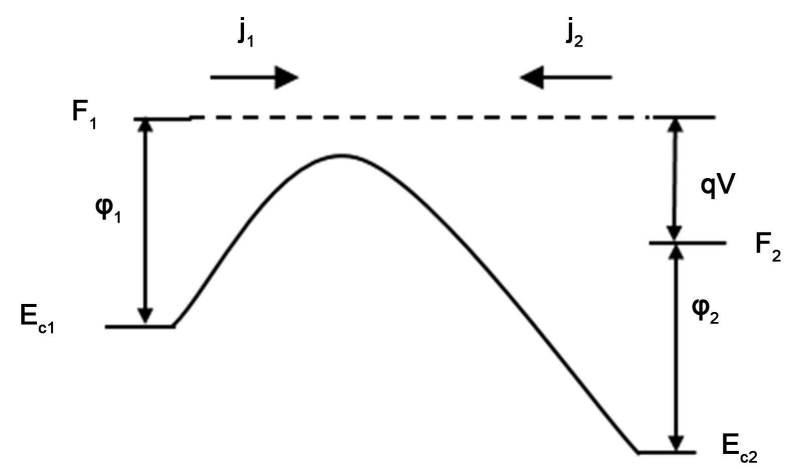

Figure 2. Change in energy levels under the external voltage. 


$$
d-x_{m}=\frac{d}{2}+\frac{\varepsilon \varepsilon_{0}(\Delta \varphi+V)}{q^{2} N_{a} d}
$$

where $\Delta \varphi=\varphi_{2}-\varphi_{1}$.

If both barriers are identical, then $\varphi_{2}-\varphi_{1}=0$. Consequently:

$$
\begin{aligned}
& d-x_{m}=\frac{d}{2}+\frac{\varepsilon \varepsilon_{0} V}{q^{2} N_{a} d} \\
& x_{m}=\frac{d}{2}-\frac{\varepsilon \varepsilon_{0} V}{q^{2} N_{a} d}
\end{aligned}
$$

With the help of Equation (5), we can determine the modulation depths of depleted layers, and the position of $x_{m}$. Using, $\Delta x_{m}=x_{m 2}-x_{m 1}$, we can determine the wavelength and the intensity of the absorbing radiation.

For that, we first determine the total photocurrent flowing through the structure. In the investigated photodetectors, the photocurrent has diffusion and drift components. To determine the diffusion photocurrent, it is necessary to find $p_{n}$ density of minority charge carriers-holes in the $n$-semiconductor. For that, the following one dimensional diffusion equation is solved [18],

$$
\frac{\partial^{2} p_{n}}{\partial x^{2}}-\frac{p_{n}}{L_{p}^{2}}=-\frac{p_{n 0}}{L_{p}^{2}}-\frac{G(x)}{D_{p}}
$$

where $L_{p}=\sqrt{D_{p} \tau_{p}}$ is the diffusion length of acceptors in the $\mathrm{n}^{+}$-region, $G(x)=F_{0} \alpha e^{-\alpha x}$ is the generation rate of electron-hole pairs, $\mathrm{F}_{0}$ represents the total flux of incident photons, $\alpha$ represents the absorption coefficient of electromagnetic radiation, $D_{p}$ is the diffusion coefficient of acceptors in the $\mathrm{n}^{+}$-region, $\tau_{p}$ the lifetime of excess charge carrier-acceptors and $\mathrm{p}_{\mathrm{n} 0}$ represents the equilibrium density of acceptors in the $n$-region. The solution of Equation (6) under the boundary conditions $p_{n}=p_{n 0}$ at $x=\infty$ and $p_{n}=0$ at $x=d\left(p_{n}\right.$-is the concentration of minority charge carriers in the rear $\mathrm{n}^{+}$-region) is

$$
I_{\text {diff }}=S\left(q p_{n 0} \frac{D_{p}}{L_{p}}+q F_{0} \frac{\alpha L_{p}}{1+\alpha L_{p}} e^{-\alpha d}\right)
$$

Khudaverdyan et al. [19] obtained an expression for the drift currents generated in the base of such structures:

$$
\begin{gathered}
I_{d r 1}=q F_{0} S\left(1-e^{-\alpha x_{m}}\right) \\
I_{d r 2}=q F_{0} S\left(e^{-\alpha x_{m}}-e^{-\alpha d}\right)
\end{gathered}
$$

where $\alpha$ is the absorption coefficient of the electromagnetic radiation, $S$-the photosensitive area and $F_{0}=P_{o p t}(1-R) / S h v$-the total flux of incident photons per unit area. $P_{\text {opt }}$-the radiation power, $R$-the reflection coefficient, $h-$ Planck constant, $v$-the frequency of the electromagnetic radiation and $q-$ the electron charge.

Taking into consideration Equations (8), (9) and (7), the expression for the total current flowing through the structure is: 


$$
I_{\text {tot }}=I_{d r 1}-I_{d r 2}-I_{d i f f}=S\left[q F_{0}\left(1-2 e^{-\alpha x_{m}}+\frac{e^{-\alpha d}}{1+\alpha L_{p}}\right)-q n_{p 0} \frac{D_{p}}{L_{p}}\right]
$$

At normal functioning, the term that contains $n_{p 0}$ is much smaller than the first term. Thus, it may be neglected, and Equation (10) is:

$$
I_{\text {tot }}=q S F_{0}\left(1-2 e^{-\alpha x_{m}}+\frac{e^{-\alpha d}}{1+\alpha L_{p}}\right)
$$

When irradiated by the integral flux (e.g. of the Sun), Equation (11) for the photocurrent is:

$$
\begin{aligned}
& \sum_{i, j} I_{P h i, j}=\sum_{i, j} I_{d r 1 i, j}-\sum_{i, j} I_{d r 2 i, j}-\sum_{i, j} I_{d i f i, j} \\
& =S q \sum_{i, j} F\left(\lambda_{i}\right)\left(1-2 e^{\alpha_{i} x_{m j}}+\frac{e^{-\alpha_{i} d}}{1+\alpha_{i} w}\right)
\end{aligned}
$$

where $(i=1,2,3, \ldots)$ changes in the integral flux with the change of the emission wavelength, and $(j=1,2,3, \ldots)$ changes with the change of bias voltage, $F_{0}\left(\lambda_{i}\right)$ is the total flux of incident photons with the wavelength $\lambda_{i}$.

Since the width of the n-region in Equation (11) was less than $L_{p}$, the value of $L_{p}$ in Equation (12) was replaced by $W$ (Figure 1). Using algorithm described below, it is possible to register a separate wavelength $\lambda_{i}$ and its intensity by changing the voltage $\Delta V$, thus obtaining the spectral dependence for wave intensities.

Let us consider some peculiarities of detection of separate waves in the integral flux of radiation using structure containing oppositely directed potential barriers. Such scenarios are used for detection of certain wavelength and its intensity under the conditions of the wave absorption and emission (fluorescence) of the objects under investigation.

In the present investigation, we used the AM0 spectral distribution for Si using radiation of LED (L-813SRC-J4) with a dominant wavelength $660 \mathrm{~nm}$ and a laser with the radiation wavelength of $530 \mathrm{~nm}$. Since, with increase of the bias voltage, the registration region of the rear barrier widens towards the surface [19] [20], it allows us to carry out the sequential registration of the waves from depth to the surface.

\section{Result of the Experiment}

To determine the spectral composition of an integral flux of the electromagnetic radiation, the wave intensities of the radiation are equally divided so that the absorption depth corresponds to the width of the registering environment ( 4 $6 \mu \mathrm{m})$. The photocurrent corresponding to the largest possible value of $x$ is normalized by the most deeply penetrated wave. Under these conditions, the process of the selection of separate waves and their intensities from the integral flux of radiation can be described as in the following.

Assume that the leading indicator signal is the photocurrent. We also assume that the largest values of $x_{m 1}$ and $x_{m 2}$ of $x_{m}$ correspond to the change of bias vol- 
tage $1 \mathrm{mV}$, and the relevant photocurrents $I_{1}$ and $I_{2}$. They are obtained only from the absorption of the most deeply penetrated wave $\lambda_{i}$. With the help of these photocurrents, and using Lambert's law on radiation absorption in the homogeneous environment, we will have the coefficient of wave absorption,

$$
\alpha_{i}=\frac{1}{\Delta x} \ln \frac{I_{2}}{I_{1}},
$$

where $\Delta x=x_{m 2}-x_{m 1}$.

Using $\alpha=f\left(\lambda_{i}\right)$ and Equation (13) with the corresponding program, we can determine the length of the wave for the initial material of the photodetector, e.g. silicon. By means of the formula for the photocurrent (11), we can obtain the intensities of separate waves in the absorbed radiation as,

$$
F_{0 i}=\frac{I_{i}}{q S\left(1-2 e^{-\alpha_{i} x_{m_{j}}}+\frac{e^{-\alpha_{i} d}}{1+\alpha_{i} w}\right)}
$$

With the help of Equations (5), (13) and (14), we determine the absorption coefficient of the most deeply penetrated wave, the wavelength and the intensity of the wave. Then, with the help of the expression (12), considering the absorption surface, we form the dependence $I_{1}=f(V)$ and subtract it from the experimental dependence $I=f(V)$ of the summed current. As a result, we obtain a new dependence of the summed photocurrent on $V$, without the dependence $I_{1}=$ $f(V)$.

By means of relevant software, this method helps us determine the lengths and the intensities of all the waves in the radiation. The experiments were carried out on the $\mathrm{n}^{+}-\mathrm{p}-\mathrm{n}^{+}$structure. The cross-section of the structure with the parameters of the layers is presented in Figure 3. The spectral region of the monochromator is from $400 \mathrm{~nm}$ up to $1150 \mathrm{~nm}$. Based on these parameters, the design height of the rear barrier was $\varphi_{K}=0.89 \mathrm{eV}$, and the distance of Fermi level from the valence band top of the p-epitaxial layer was $E_{F}-E_{V}=0.29 \mathrm{eV}$ (Figure 4). The height of the silicide Schottky barrier (p-silicon with Ag) was $0.54 \mathrm{eV}$ [18], and, consequently, the height of the surface barrier was $\phi_{c}=0.25 \mathrm{eV}$.

The photocurrents of the two oppositely directed barriers are oppositely directed, and they compensate each other depending on the applied voltage. It is noticeable in the relative spectral characteristics (Figure 5). The figure shows the mirror image of the long wave photocurrent. In the absence of the bias voltage (Figure 5, curve. 1), the spectral photocurrent is mainly governed by the surface potential barrier, which creates a short-wave peak.

The long-wave peak has small height and is governed by the rear barrier, which is located at the depth of $6 \mu \mathrm{m}$ from the illuminated surface (Figure 3). When the bias voltage is " + " on the Schottky contact, the surface barrier is shifted in the reverse direction, and the barrier of the p-n junction is shifted in the forward direction, which increases the photocurrent of the first barrier (Figure 5, Curve 2). When the bias voltage sign is “-”, the rear barrier is shifted in the reverse direction and the spectral photocurrent is governed by its increase 


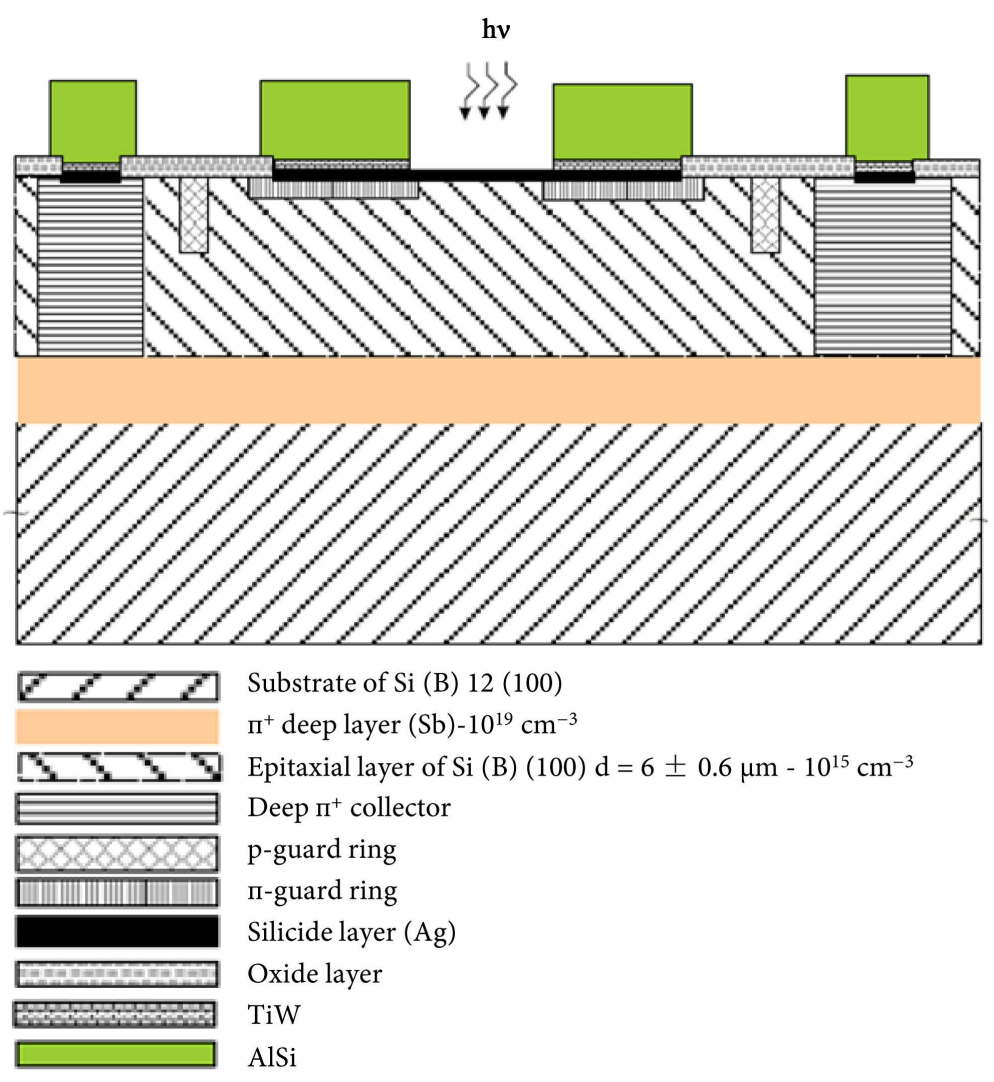

Figure 3. Cross-section of the structure and the parameters of individual layers.

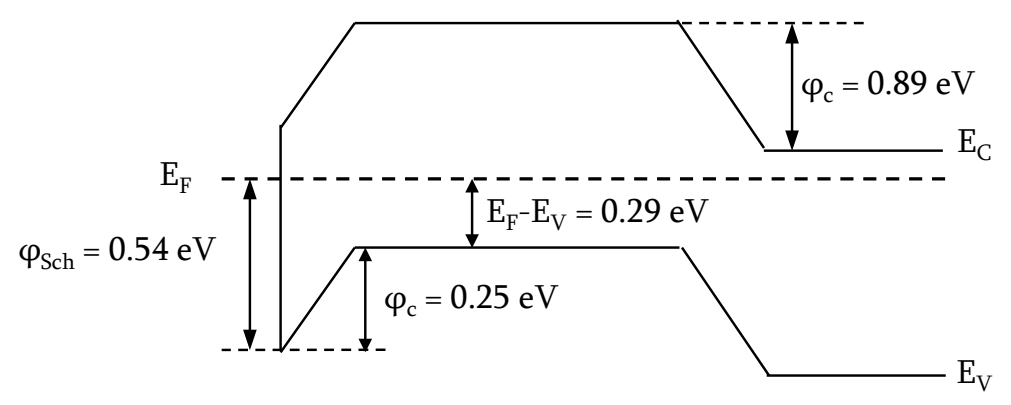

Figure 4. Energy band diagram of the structure.

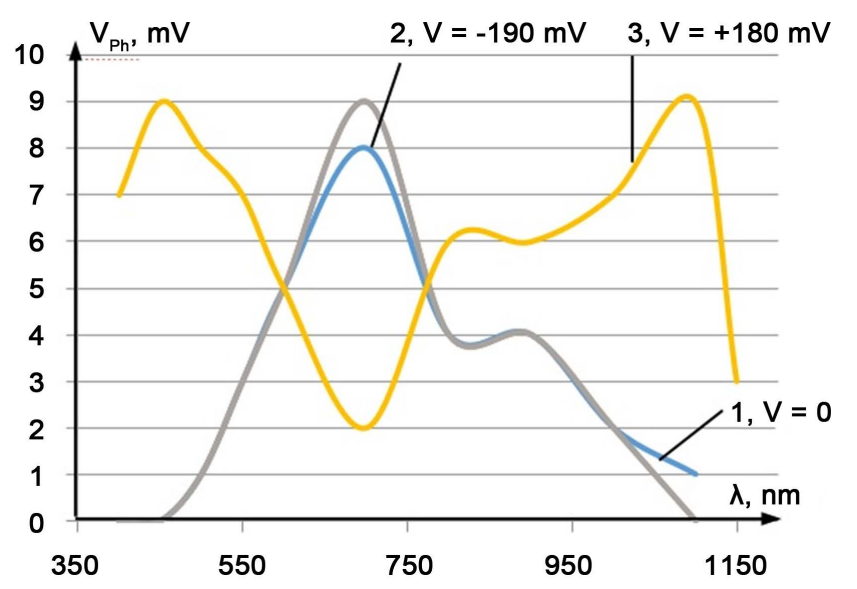

Figure 5. Spectral distribution of the photovoltage on the load resistor of $0.5 \mathrm{M} \Omega$. 
(Figure 5, Curve 3), and the long-wave peak becomes more noticeable. Such mutual compensation of the photocurrents of both barriers is related to the change in the width of the wave registration region.

Thus, at different voltages, different waves penetrate into the registration region of the rear barrier. These waves determined by the corresponding change of the photocurrent. During the experiment, for determining the spectral distribution of the intensity of the absorbed radiation, the L-813SRC-J4 LEDs and laser beam (wavelength of $530 \mathrm{~nm}$ ) were used. The experimental current-voltage characteristic in the dark (Figure 6) showed that in the wide range of the bias voltage, the currents had small values (several dozens of $\mathrm{pA}$ ). The photocurrents had the values of several $\mu \mathrm{A}$ (Figure 7). Several orders of the difference of light and dark currents allowed us to register low fluxes of light signals with the help of the test samples.

The design width of the depleted layer of the $n-p$ junction under the zero bias is $\sim 1 \mu \mathrm{m}$, and $2.55 \mu \mathrm{m}$ under the reverse voltage of $\sim 5 \mathrm{~V}$. Thus, we observe the modulation of the width of the registration region of the rear barrier. With the help of Equation (13) and (14), the current-voltage characteristic data in the dark and under illumination, and the developed algorithm [21], we modelled the process of receiving of the spectral distribution of the radiation intensity. As a result, the received spectral curve (Figure 8 ) is close to the stated curve of the red LED and the laser with the radiation wavelength of $530 \mathrm{~nm}$ (Figure 9).

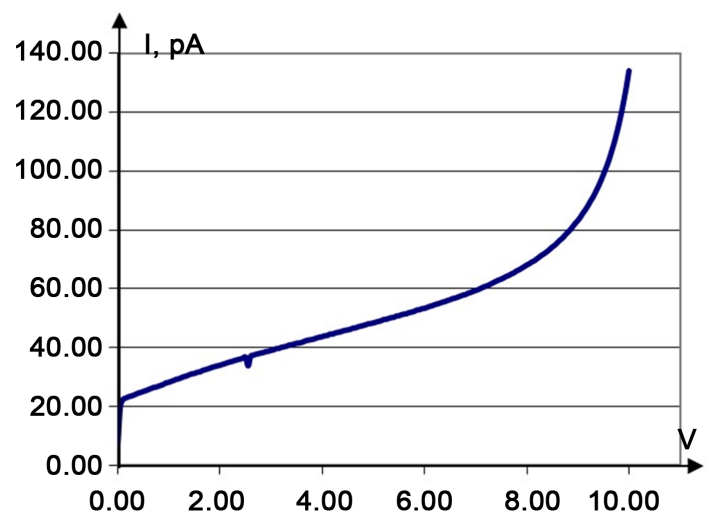

Figure 6. Voltage-current characteristic of the sample in the dark.

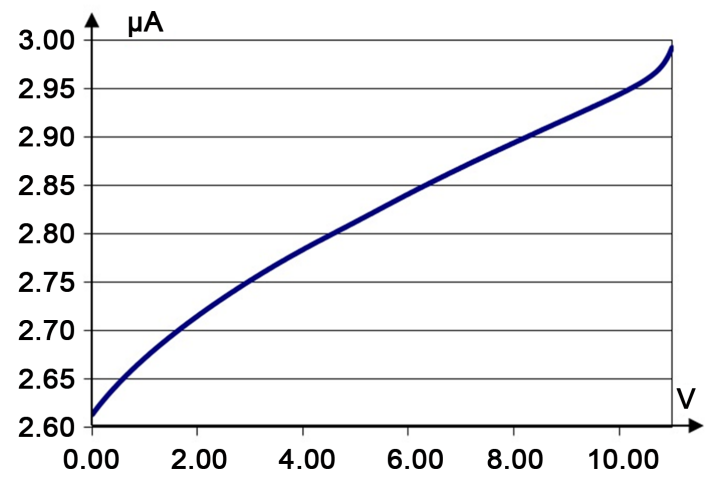

Figure 7. Voltage-current characteristic of the sample under illumination. 


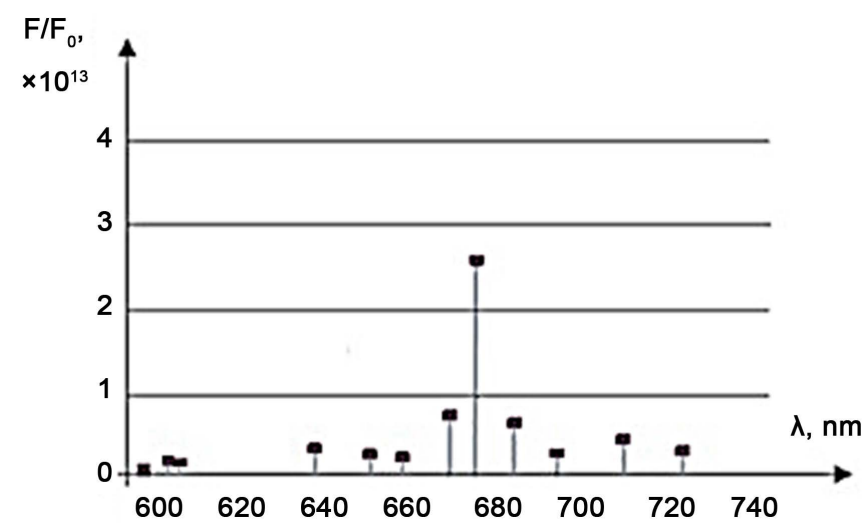

Figure 8. Spectral distribution of radiation intensity.

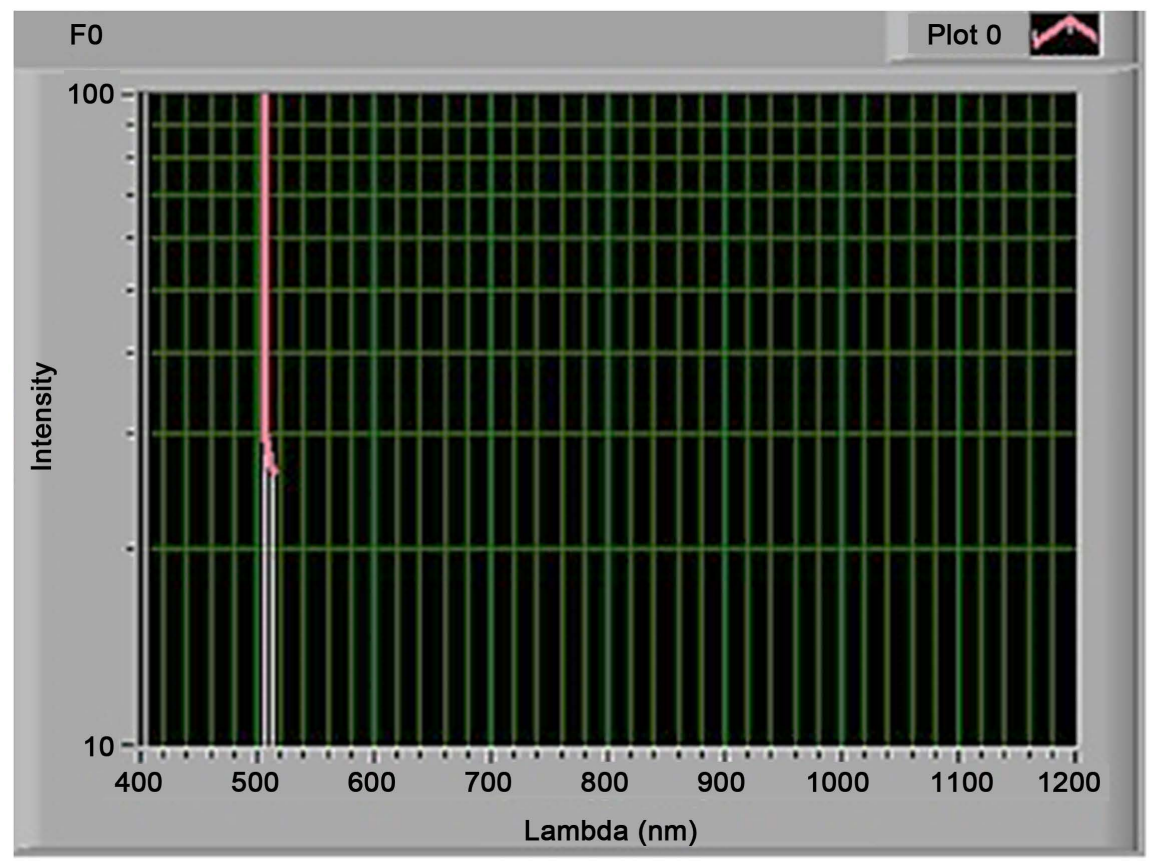

Figure 9. The spectral distribution of the laser wave.

\section{Conclusions}

- We have developed a new model of the portable photosensor for the remote identification of optical information. Different absorption depths of electromagnetic waves in the developed silicon photodetector are used to create an algorithm for the determination of the wavelength and its intensity.

- Experimental samples have been investigated and possibility of selective spectral sensitivity in them has been proven. That is possible thanks to variation of potential barriers in bases of the samples at the expense of each other when applying external voltage.

- Spectral distributions of intensities of red LED and the laser with the radiation wavelength of $530 \mathrm{~nm}$ have been received using the developed algorithm. It repeats the reference distribution, with certain approximation.

- The results of the testing of the algorithm on the experimental samples open 
new possibilities for the creation of a new type of the portable semiconductor spectrophotometer. The developed algorithm is used to carry out the spectral analysis without a preliminary calibration.

\section{Acknowledgements}

The authors express their gratitude to the company "RD ALFA Microelectronics" for their assistance in the prototyping of the sample photodetectors. We also acknowledge support of NATO Science for Peace and Security-G4403 for partial support of this investigation.

\section{References}

[1] Jiang, P., Xia, H.B., He, Z.Y. and Wang, Z.M. (2009) Design of a Water Environment Monitoring System Based on Wireless Sensor Networks. Sensors, 9, 64116434. https://doi.org/10.3390/s90806411

[2] Normatov, P.I., Armstrong, R., Normatov, I.Sh. and Narzulloev, N. (2015) Monitoring Extreme Water Factors and Studying the Anthropogenic Load of Industrial Objects on Water Quality in the Zeravshan River Basin. Russian Meteorology and Hydrology, 40, 347-354. https://doi.org/10.3103/S106837391505009X

[3] http://augsignals.com/products-services/water-quality-monitoring/

[4] Vaseashta, A. (2015) Life Cycle Analysis of Nanoparticles-Risk, Assessment, and Sustainability. Destech Publishers, New York, 404 p.

[5] Vaseashta, A., Braman, E., Susmann, P., Dekhtyar, Yu. and Perovicha, K. (2012) Sensors for Water Safety and Security. Surface Engineering and Applied Electrochemistry, 48, 478-486.

[6] Pshinko, G.N., Kobets, S.A. and Puzyrnayain, L.N. (2013) Concentration of U(VI) on a Complexing Sorbent for Its Determination by the Spectrophotometric Method. Journal of Water Chemistry and Technology, 35, 145-151. https://doi.org/10.3103/S1063455X13040012

[7] http://www.shimadzu.com/an/uv/support/fundamentals/structure.html

[8] http://www.dissercat.com/content/razrabotka-i-primenenie-distantsionnykh-spektr ometricheskikh-metodov-issledovaniya-prirodnyk

[9] Kautzsch, Th. (2013) Photo Cell Devices and Methods for Spectrometric Applications. Patent US 20130285187 A1.

[10] Kautzsch, Th. (2014) Photodetector with Controllable Spectral Response. Patent US 8916873 B2.

[11] Kautzsch, T. (2012) Photocell Devices and Methods for Spectroscopic Applications. Patent DE 102013207801 A1.

[12] Jan, C., Daniel, P., Man, S.T. and Univ, N. (2005) Photodetector with Controllablespectral Response. Patent US 8916873 B2.

[13] Nataša, G. and Novel, A. (2007) Type of Tri-Colour Light-Emitting-Diode-Based Spectrometric Detector for Low-Budget Flow-Injection Analysis. Sensors, 7, 166184. https://doi.org/10.3390/s7020166

[14] Elif, Ç.S., David, S.F., Mutlu, G., Ekmel, Z., Mesut, S. and Selim, M. (2014) Improved Selectivity from a Wavelength Addressable Device for Wireless Stimulation of Neural Tissue. Frontiers in Neuroengineering, 7, 5.

[15] Nader, M.K. and Fereydoon, N. (2004) Filterless Si-Based Ultraviolet-Selective Photodetectors. Spire Corp. for Stennis Space Center, Mississippi, Refer to SSC- 
00072.

[16] Vanyushin, I.V., Gergel, V.A., Zimoglyad, V.A. and Tishin, Y.I. (2005) Adjusting the Spectral Response of Silicon Photodiodes by Additional Dopant Implantation. Russian Microelectronics, 34, 155-159. https://doi.org/10.1007/s11180-005-0024-5

[17] Gergel, V.A., Lependin, A.V., Tishin, Y.I., et al. (2006) Boron Distribution Profiling in Asymmetrical NC-P Silicon Photodiodes and New Creation Concept of Selectively Sensitive Photoelements for Megapixel Color Photoreceivers.

[18] Sze, S. (1981) Physics of Semiconductor Divices. 2nd Edition, Wiley, New York, 450.

[19] Khudaverdyan, S., Petrosyan, O., Dokholyan, J., Khudaverdyan, D. and Tsaturyan, S. (2012) Modeling of a New Type of an Optoelectronic Biosensor for the Monitoring of the Environment and the Food Products. In: Vaseashta, A., Braman, E. and Susmann, P., Eds., Technological Innovations in Sensing and Detection of Chemical, Biological, Radiological, Nuclear Threats and Ecological Terrorism, Springer, Berlin, 179-184. https://doi.org/10.1007/978-94-007-2488-4_17

[20] http://rredc.nrel.gov/solar/spectra/am0/wehrli1985.new.html

[21] Khudaverdyan, S., Khachatryan, M., Khudaverdyan, D., Tsaturyan, S. and Vaseashta, A. (2013) New Model of Spectral Analysis of Integral Flux of Radiation. In: Vaseashta, A. and Khudaverdyan, S., Eds., Advanced Sensors for Safety and Security, Springer, Berlin, 261-269. https://doi.org/10.1007/978-94-007-7003-4_23

Submit or recommend next manuscript to SCIRP and we will provide best service for you:

Accepting pre-submission inquiries through Email, Facebook, LinkedIn, Twitter, etc. A wide selection of journals (inclusive of 9 subjects, more than 200 journals) Providing 24-hour high-quality service User-friendly online submission system Fair and swift peer-review system Efficient typesetting and proofreading procedure Display of the result of downloads and visits, as well as the number of cited articles Maximum dissemination of your research work

Submit your manuscript at: http://papersubmission.scirp.org/

Or contactopj@scirp.org 